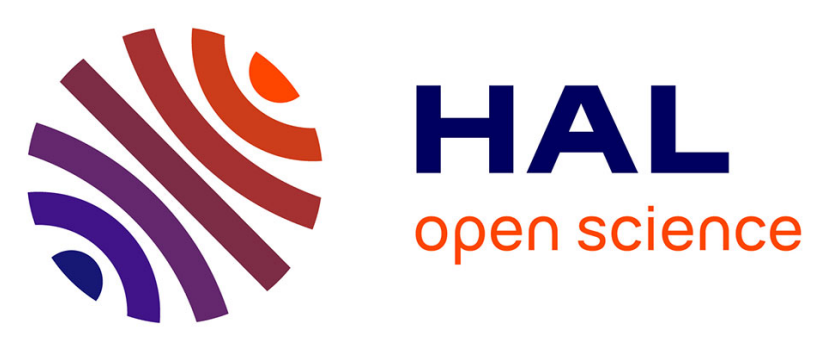

\title{
Oriented Metallic Nano-Objects on Crystalline Surfaces by Solution Epitaxial Growth
}

Nikolaos Liakakos, Charbel Achkar, Benoit Cormary, Justine Harmel, Bénédicte Warot-Fonrose, Etienne Snoeck, Bruno Chaudret, Marc Respaud, Aikaterini Soulantika, Thomas Blon

\section{To cite this version:}

Nikolaos Liakakos, Charbel Achkar, Benoit Cormary, Justine Harmel, Bénédicte Warot-Fonrose, et al.. Oriented Metallic Nano-Objects on Crystalline Surfaces by Solution Epitaxial Growth. ACS Nano, 2015, 9 (10), pp.9665 - 9677. 10.1021/acsnano.5b04524 . hal-01707151

\section{HAL Id: hal-01707151 \\ https://hal.science/hal-01707151}

Submitted on 7 Mar 2018

HAL is a multi-disciplinary open access archive for the deposit and dissemination of scientific research documents, whether they are published or not. The documents may come from teaching and research institutions in France or abroad, or from public or private research centers.
L'archive ouverte pluridisciplinaire HAL, est destinée au dépôt et à la diffusion de documents scientifiques de niveau recherche, publiés ou non, émanant des établissements d'enseignement et de recherche français ou étrangers, des laboratoires publics ou privés. 


\title{
Oriented Metallic Nano-Objects on Crystalline
}

\section{Surfaces by Solution Epitaxial Growth}

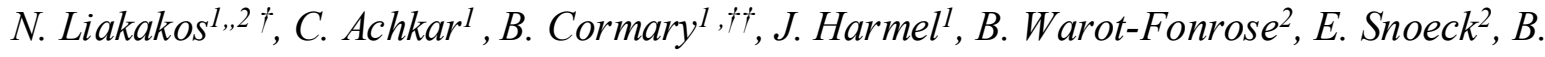 \\ Chaudret $^{1}$, M. Respaud ${ }^{1}$, K. Soulantica ${ }^{1, *}$, T. Blon ${ }^{1, *}$
}

${ }^{1}$ Laboratoire de Physique et Chimie des Nano-Objets, LPCNO, UMR5215 INSA-UPS-CNRS, Université de Toulouse; Institut National des Sciences Appliquées, 135 avenue de Rangueil, 31077 Toulouse, France

${ }^{2}$ Centre d'Elaboration de Matériaux et d'Etudes Structurales (CEMES-CNRS), 29 rue Jeanne Marvig, B.P. 94347, 31055 Toulouse, France

ABSTRACT. Chemical methods offer the possibility to synthesize a large panel of nanostructures of various materials with promising properties. One of the main limitations to a mass market development of nanostructure based devices is the integration at a moderate cost of nano-objects into smart architectures. Here we develop a general approach by adapting the seedmediated solution phase synthesis of nanocrystals in order to directly grow them on crystalline thin films. Using a Co precursor, single-crystalline Co nanowires are directly grown on metallic films and present different spatial orientations depending on the crystalline symmetry of the film used as a 2D seed for Co nucleation. Using films exposing 6-fold symmetry surfaces such as 
$\operatorname{Pt}(111), \operatorname{Au}(111)$ and $\mathrm{Co}(0001)$, the Co heterogeneous nucleation and epitaxial growth leads to vertical nanowires self-organized in dense and large scale arrays. On the other hand, using films presenting 4-fold symmetry surfaces such as $\mathrm{Pt}(001)$ and $\mathrm{Cu}(001)$, the Co growth leads to slanted wires in discrete directions. The generality of the concept is demonstrated with the use of a Fe precursor which results in $\mathrm{Fe}$ nanostructures on metallic films with different growth orientations which depend on the 6-fold/4-fold symmetry of the film. This approach of solution epitaxial growth combines the advantages of chemistry in solution in producing, shape-controlled and mono-disperse metallic nanocrystals, and of seeded growth on an ad-hoc metallic film that efficiently controls orientation through epitaxy. It opens attractive opportunities for the integration of nanocrystals in planar devices.

KEYWORDS: epitaxial growth, seeded growth, self-organization, cobalt nanowire, iron nanoparticle, nanowire array.

Solution phase synthesis of nanoparticles can produce size, shape and composition controlled nanocrystals of almost all kind of materials (semiconducting, metallic, metal oxides), possessing remarkable properties (optical, sensing, catalytic, magnetic, etc). Despite these promising properties, nanocrystal implementation in real applications faces several problems. Indeed, a great number of targeted nanostructure-based devices, especially in the domain of microelectronics, require the exact positioning and orientation of nano-scale building blocks locally, or over extended areas. ${ }^{1}$ The organized integration of these nano-objects produced by chemical methods into future functional devices that will exploit their specific properties is in the 
center of a very active area of research. ${ }^{2-5}$ In comparison to top-down approaches, bottom-up processes are expected to organize nanostructures over large scales with a reduced number of technological steps. ${ }^{6-8}$ Self-assembly of colloidal nanocrystals offers a low-cost solution to largescale fabrication of ordered nanoscale materials. ${ }^{9}$ It is however inappropriate for controlling nano-crystal orientation with respect to a substrate. Moreover, the chemical and mechanical stability of the architecture is a prerequisite for future applications to ensure reliable and stable properties over time. ${ }^{10}$ Finally, rendering easier an efficient electrical contact between macroscopic components and the nano-object is of utmost importance for optoelectronic applications. ${ }^{11}$

An attractive alternative is based on the oriented growth of nanocrystals directly on surfaces. Considering 1D nanocrystals, several bottom-up methods for growing nanowires on surfaces have been developed, especially the well-known vapor-liquid-solid (VLS) technique ${ }^{12}$ and its derivatives. ${ }^{13}$ Thanks to a metallic catalyst deposited on a surface and initiating the growth, the VLS method leads to vertical or tilted epitaxial growth of nanowires on substrates. ${ }^{14,15}$ However, such a process allows to produce almost exclusively semiconducting nanostructures. Recently, a lot of effort has been devoted to the development of aqueous routes for growing $\mathrm{Fe}_{2} \mathrm{O}_{3}, \mathrm{Mn}_{2} \mathrm{O}_{3}$, $\mathrm{TiO}_{2}, \mathrm{ZnO}$ nanorods (NRs) / nanowires (NWs) perpendicular to a substrate without using any rigid template or catalyst. ${ }^{16}$ These NRs/NWs are at the origin of intensive research, due to their high potential for numerous applications (photovoltaic devices, gas sensors, etc), and their integration into devices is an intense area of investigation. ${ }^{17}$ Considering 1D metallic nanocrystals, arrays of vertical metallic nanowires can be obtained by filling mesoporous templates such as anodized aluminum oxide ${ }^{18,19}$ or copolymer templates ${ }^{20}$ by chemical, electrochemical or vacuum deposition techniques..$^{21,22}$ The nanowire diameter is controlled by the 
pore diameter which is generally larger than 10-12 nm, even if diameters as small as $8 \mathrm{~nm}$ can be attained. ${ }^{23}$ The main advantage of electrochemical methods is their ability to deposit a wide variety of materials depending on the plating bath used. Nevertheless, in the majority of the cases, the structure of the resulting wires is polycrystalline, and crystallite orientation is poorly controlled. ${ }^{24}$ Nanowires or nano-columns stabilized into crystalline matrices have also been reported, ${ }^{25-27}$ as well as Co NWs directly grown by chemical vapor deposition (CVD) without templates ${ }^{28}$ but they also suffer from poor crystalline order and low density respectively. Until very recently there was no pure bottom-up approach that leads to the oriented growth of sub10nm single-crystalline metallic nanowires on surfaces without the use of templates or matrices. The potential applications of arrays of metallic nanowires on surfaces is however important, for instance in sensing and metamaterials ${ }^{29,30}$ considering noble metals, or in ultra-high density magnetic recording using magnetic materials. ${ }^{20,31}$ In this context, colloidal chemistry is particularly attractive as it can produce well controlled 1D nano-objects with good crystalline order. $^{32,33}$ Moreover, seed-mediated growth of nanocrystals in solution has been extensively studied using nanometer size seeds. This strategy allows the synthesis of a variety of complex hybrid nano-structures, among which bimetallic and multicomponent nanocrystals. ${ }^{34-36}$ Homo- or hetero-epitaxial relationships between the seed and the growing material have been used in order to control the shape of the final nanostructures. ${ }^{37,38}$ However, this solution approach has not been largely applied to macroscopic seeds for the direct elaboration of metallic nanostructures. We recently reported the adaptation of a solution synthesis of Co nanorods, that allows to epitaxially grow single-crystalline cobalt nanowires on a $2 \mathrm{D}$ non-patterned $\mathrm{Pt}(111)$ metallic film. ${ }^{39}$ The nanowire growth starts from the metallic film immersed in solutions of compositions similar to the ones that, in the absence of any seed film, give rise to anisotropic Co nano-objects. ${ }^{40,41}$ This 
strategy combines the major advantages of both chemical and physical methods: (i) the possibility of decomposing metal salts, coordination compounds, or organo-metallic precursors in solution to obtain, shape-controlled and mono dispersed sub-10 nm nanocrystals, and (ii) the epitaxial growth of nanocrystals on an ad-hoc crystalline film. Here we demonstrate that this epitaxial growth of Co nanowires is also successful on seed layers different from $\operatorname{Pt}(111)$, namely $\mathrm{Co}(0001), \mathrm{Au}(111), \mathrm{Pt}(001)$ and $\mathrm{Cu}(001)$. The solution composition imposes anisotropic Co growth on all these substrates which differ from each other, either in chemical composition, in symmetry, or in lattice parameters. We show by a precise crystallographic analysis that the crystalline symmetry of the seed layer has a strong impact on the heterogeneous nucleation of cobalt, on its crystalline growth direction and therefore on the nanowire orientations. Consequently, by acting on the nature of the metallic film introduced into the solution synthesis, vertical or tilted nanowires with discrete angles with respect to the substrate can be obtained. Furthermore we demonstrate the generality of this approach of solution epitaxial growth, showing how by decomposition of a Fe precursor, Fe nanostructures of different orientations depending on the crystalline symmetry of the film, can be grown on metallic films. This study establishes that the solution epitaxial growth constitutes a novel approach for the direct growth of nanocrystals with tunable growth orientations, shape control and epitaxy on planar devices.

\section{RESULTS}

Three kinds of films are compared as seed layer: (i) films with crystalline planes of 6-fold symmetry, $\operatorname{Pt}(111), \operatorname{Au}(111)$ and $\operatorname{Co}(0001)$, (ii) films with planes of 4-fold symmetry, $\operatorname{Pt}(001)$ and $\mathrm{Cu}(001)$, and (iii) commercial polycrystalline $\mathrm{Cu}$ metal foils. Apart from the commercial substrates, the films were grown epitaxially by sputtering and/or thermal evaporation of the 
corresponding metal target (i) on $\mathrm{Al}_{2} \mathrm{O}_{3}(0001)$ or $\mathrm{Si}(001)$ substrates for 6-fold symmetry crystalline surfaces, and (ii) on $\mathrm{MgO}(001)$ substrates for the 4-fold symmetry substrates (see procedure description and elaboration conditions in Methods section and Supporting Table S1 respectively). In a second step, the substrate is introduced into an anisole or toluene solution of $\left[\mathrm{Co}\left\{\mathrm{N}\left(\mathrm{SiMe}_{3}\right)_{2}\right\}_{2}(\mathrm{thf})\right]$ containing lauric acid (LA) and hexadecylamine (HDA). The solution containing the substrate supporting the metallic film is then heated, under 3 bars of $\mathrm{H}_{2}$. It has to be noted that, as a result of homogeneous nucleation, free Co nano-objects are formed at $150^{\circ} \mathrm{C}$ and with concentrated solutions $\left(50 \mathrm{mM}\right.$ in Co) $\cdot^{40,41}$ Thus at $[\mathrm{Co}]=50 \mathrm{mM}$, and when heated under $\mathrm{H}_{2}$ at $150^{\circ} \mathrm{C}$, a molecular ratio of $1 \mathrm{Co} / 1.2 \mathrm{LA} / 1.2 \mathrm{HDA}$, in the absence of films gives rise to Co nanorods, ${ }^{40}$ whereas a ratio of $1 / 2 / 2$ gives rise to multipods due to the initial nucleation of irregularly shaped nanocrystals from where anisotropic pods grow during the growth step. ${ }^{41}$ Whereas the growth of free Co nano-objects in the absence of added seeds is preceded by a homogeneous nucleation in solution, the exclusive Co growth on a metallic film requires the suppression of this stage. The reaction conditions were chosen so that the formation of free Co nano-objects in solution is not favored. Therefore the temperature and/or the Co concentration have been decreased compared to the synthesis of free Co nano-objects in order to limit the homogenous nucleation and favor the heterogeneous nucleation of Co on the film. While we have reduced the concentration and/or the reaction temperature, we used Co/LA/HDA ratios that give rise to anisotropic Co nanocrystals. Using this approach, we have previously shown that a reaction of 4.5 days at $100^{\circ} \mathrm{C}$ using a $\mathrm{Co} / \mathrm{LA} / \mathrm{HDA}$ molecular ratio of $1 / 1.2 / 1.2$ and with a Co concentration of about $2 \mathrm{mM}$ results in vertical Co nanorods (NRs) of $140 \mathrm{~nm}$ in length, $8 \mathrm{~nm}$ in diameter and epitaxially grown on $\operatorname{Pt}(111)$. Using the second ratio of $1 / 2 / 2$, a reaction of $24 \mathrm{~h}$ at $150^{\circ} \mathrm{C}$ and at concentrations of about $5 \mathrm{mM}$ results in vertical Co nanowires (NWs) on $\mathrm{Pt}(111)$ 
of $960 \mathrm{~nm}$ in length and 5-6 $\mathrm{nm}$ in diameter. ${ }^{39}$ The same solution compositions under similar reaction conditions lead to various configurations of NRs and NWs on thin films of various metals and different crystalline symmetries.

\section{Co nanowire growth on 6-fold symmetry metallic films}

Figure 1 displays the scanning electron microscope (SEM) micrographs of three different films exposing crystalline planes of 6-fold symmetry, after independent and identical $24 \mathrm{~h}$ reactions at $150^{\circ} \mathrm{C}$ using a $\mathrm{Co} / \mathrm{LA} / \mathrm{HDA}$ molecular ratio of $1 / 2 / 2$. Interestingly, the nanowires are oriented perpendicularly to the substrate whatever the metallic film, $\operatorname{Pt}(111), \operatorname{Co}(0001)$ and $\operatorname{Au}(111)$. While tilted NWs are clearly seen at the periphery of bundles, perpendicular NWs are densely packed into bundles of various sizes depending of the metallic film. Cross-section observations using transmission electron microscopy (TEM) of Co NW arrays on Pt(111) and Co(0001) films confirm the vertical growth of Co nanowires (Figure 2). A TEM cross-section of Co NWs on $\mathrm{Au}(111)$ is shown in Supporting Figure S1. Whatever the seed metallic film, the NW length is estimated to be around $1 \mu \mathrm{m}$ (Table 1). We suppose that bending and therefore bundle formation of NWs occurs due to their micrometric length because shorter NRs do not present such a bendingErreur! Signet non défini. (see also Supporting Fig. S2a). These cross-section observations reveal that the NW densities obtained for Co NWs/Pt(111) and Co NWs/Co(0001) are not as drastically different as suggested by Figure 1. Nevertheless, the differences in bundle sizes can be due to differences in film quality (grain size, roughness) of the $\operatorname{Pt}(111), \operatorname{Co}(0001)$ and $\mathrm{Au}(111)$ films (see Supporting Table S1). The NW diameters are estimated by cross-section TEM micrographs to be around $6 \mathrm{~nm}, 8 \mathrm{~nm}$ and $9 \mathrm{~nm}$ for Co NWs/Pt(111), Co NW s/Co(0001) and $\mathrm{Co} \mathrm{NWs} / \mathrm{Au}(111)$ respectively (Table 1$)$. The $\mathrm{Co}[0002]$ growth direction is also locally 
evidenced in high resolution TEM micrographs of Co NWs on $\operatorname{Pt}(111)$ and $\mathrm{Co}(0001)$ (Fig. 2c-f, respectively). It demonstrates that the Co nanowires are single-crystals with the $c$ axis of the Co bulk $h c p$ structure along the wire axis.

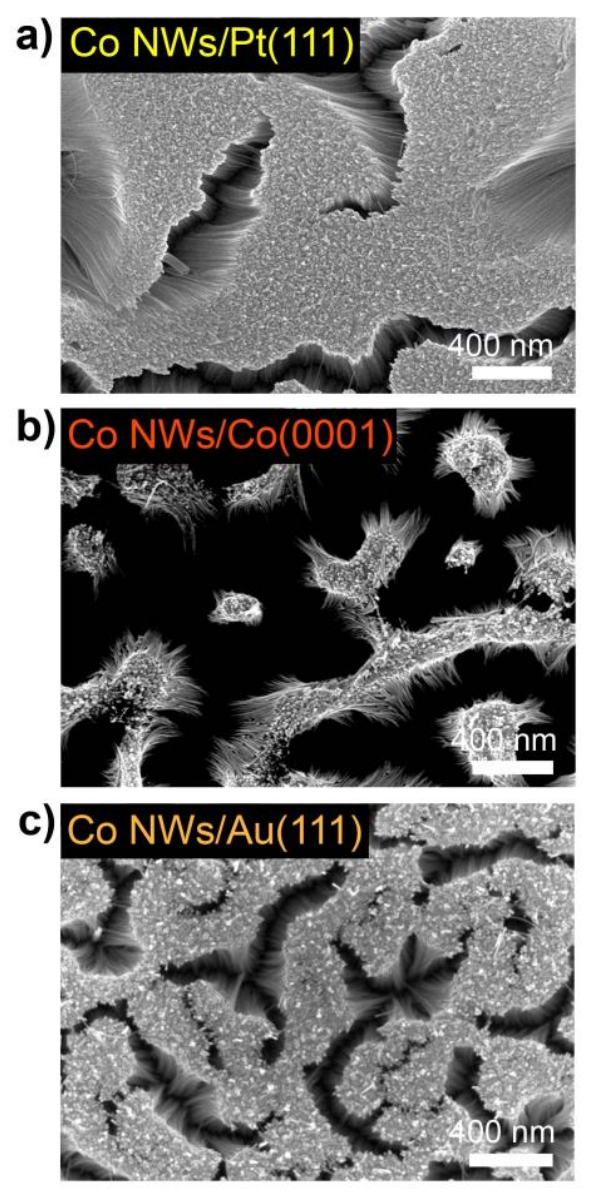

Figure 1. Scanning Electron Microscopy (SEM) micrographs of the surfaces of Co nanowire arrays grown on (a) $\mathrm{Pt}(111) / \mathrm{Al}_{2} \mathrm{O}_{3}(0001)$, (b) $\mathrm{Co}(0001) / \mathrm{Al}_{2} \mathrm{O}_{3}(0001)$ and (c) $\mathrm{Au}(111) / \mathrm{Cr} / \mathrm{Si}(001)$, after $24 \mathrm{~h}$ of reaction $\left(\mathrm{Co} / \mathrm{LA} / \mathrm{HDA}\right.$ ratio: $1 / 2 / 2,[\mathrm{Co}]=5 \mathrm{mM}, 24 \mathrm{~h}, 150^{\circ} \mathrm{C}$, solvent: anisole $)$. 

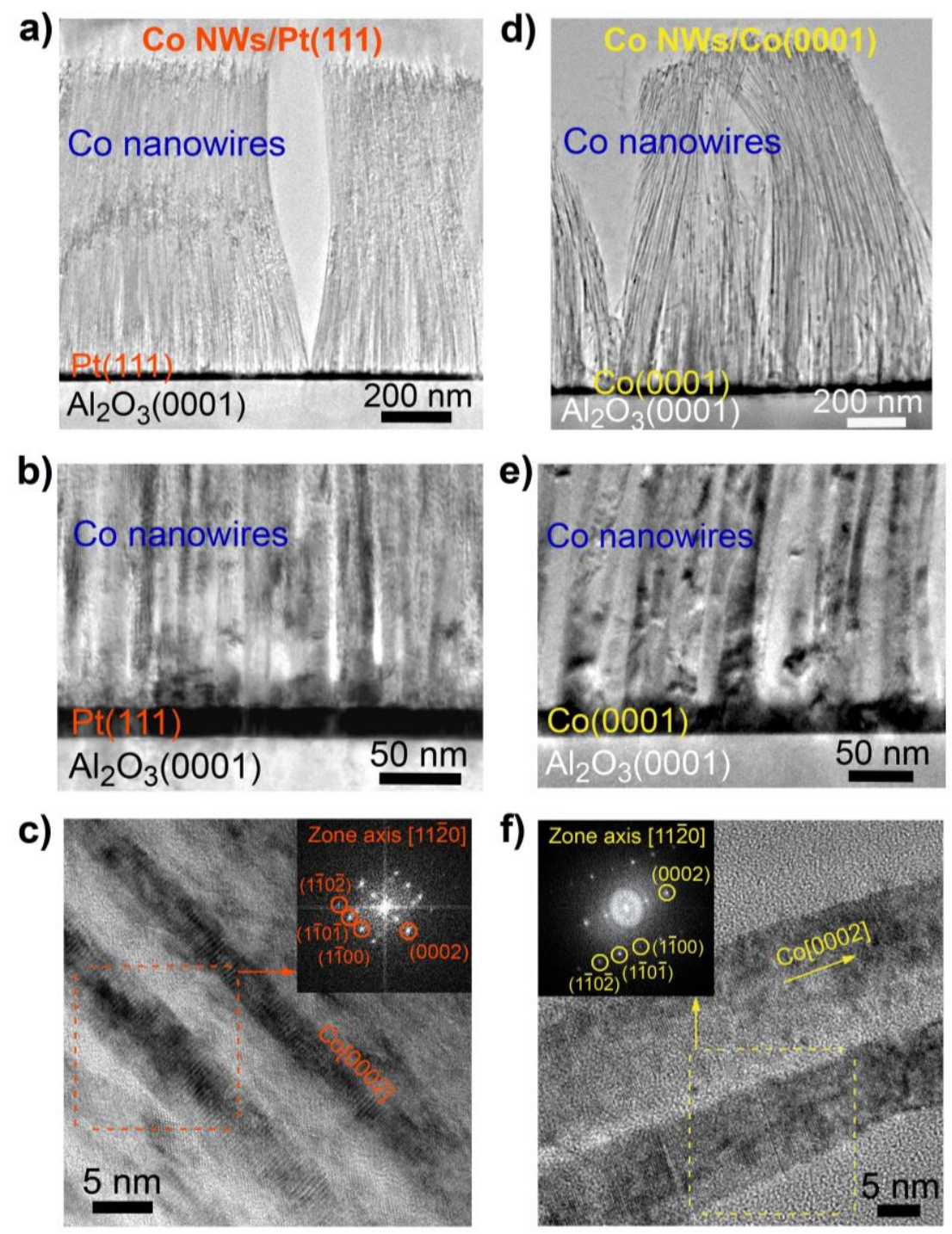

Figure 2. Cross-sectional transmission electron microscope (TEM) micrographs of Co nanowire arrays grown on (a),(b),(c) $\mathrm{Pt}(111) / \mathrm{Al}_{2} \mathrm{O}_{3}(0001)$ and (d),(e),(f) $\mathrm{Co}(0001) / \mathrm{Al}_{2} \mathrm{O}_{3}(0001)$, (Co/LA/HDA ratio: $1 / 2 / 2,[\mathrm{Co}]=5 \mathrm{mM}, 24 \mathrm{~h}, 150^{\circ} \mathrm{C}$, solvent: anisole). (c) and (f) are high resolution TEM micrographs of Co nanowires on $\mathrm{Pt}(111) / \mathrm{Al}_{2} \mathrm{O}_{3}(0001)$ and $\mathrm{Co}(0001) / \mathrm{Al}_{2} \mathrm{O}_{3}(0001)$ respectively. Insets: fast Fourier transforms illustrating the single-crystalline structure of the nanowires with a $\mathrm{Co}[0001]$ growth axis, i.e. the $c$-axis of the Co hcp structure. 
In contrast to electron microscope techniques that give information on limited sample areas, XRay diffraction (XRD) allows interrogating the sample over large areas. Therefore, it was the method of choice for the crystallographic characterization of the obtained nanostructured films. The symmetric $\theta-2 \theta$ diffractograms (Fig. 3a) indicate that the $\operatorname{Co}(0002)$ planes are parallel to the $\operatorname{Pt}(111), \operatorname{Co}(0001)$ and $\mathrm{Au}(111)$ planes, respectively (the different axes and angles relevant to the XRD measurements are shown in Figure S3). Combined to the TEM observations, these data show that the wires grow vertically along the $\mathrm{Co}[0002]$ direction whatever the metallic film of 6fold symmetry. In order to precise the NW spatial orientation, we performed XRD texture measurements to record the pole figure of the $\mathrm{Co}\{0002\}$ reflections, i.e. the NW growing planes (Figure 3b-d, and Methods section). Indeed, as NWs grow along the (0001) planes in a singlecrystal $h c p$ structure, the spatial orientation of the (0001) planes corresponds to the NW ones. The Co $\{0002\}$ pole figures recorded on NW arrays grown on 6-fold symmetry surfaces reveal that the $\mathrm{Co}[0002]$ growth direction, and consequently the direction of the NW long axis, is narrowly distributed around the substrate out-of-plane direction $\left(\chi=\varphi=0^{\circ}\right)$, even if a larger distribution is recorder for Co $\mathrm{NWs} / \mathrm{Co}(0001)$. A 3D scheme of the vertical NWs grown on 6fold symmetry surfaces is given in Figure $3 \mathrm{e}$. 

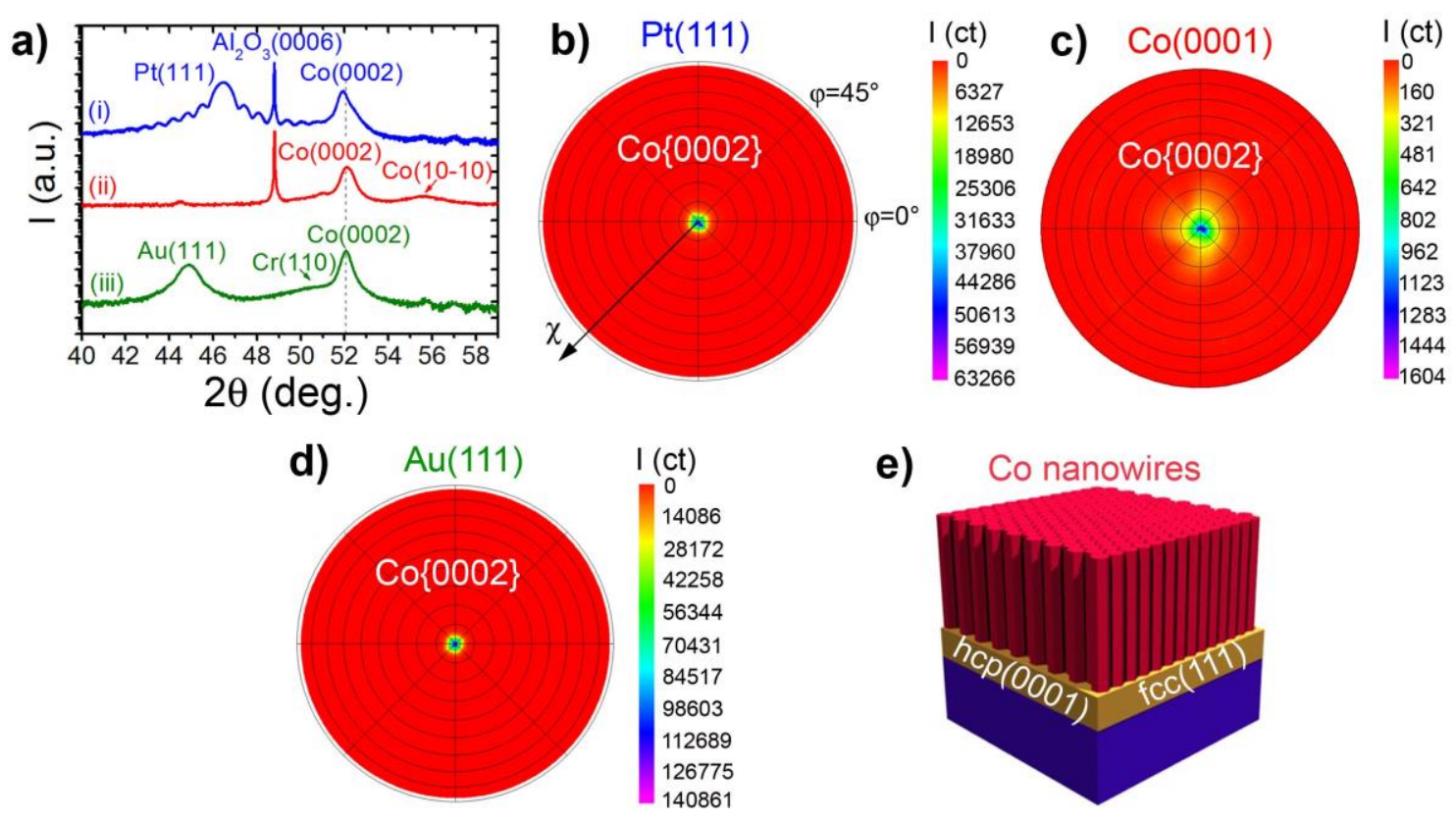

Figure 3. (a) XRD $\theta-2 \theta$ diffractograms $\left(\mathrm{Co} \mathrm{K}_{1}\right)$ of Co NW arrays grown on different metallic films: (i) $\mathrm{Pt}(111)$, (ii) $\mathrm{Co}(0001)$, and (iii) $\mathrm{Au}(111)\left(\mathrm{Co} / \mathrm{LA} / \mathrm{HDA}\right.$ ratio: $1 / 2 / 2,[\mathrm{Co}]=5 \mathrm{mM}, 24 \mathrm{~h}, 150^{\circ} \mathrm{C}$, solvent: anisole). The diffractogram measured on $\mathrm{Co} \mathrm{NWs/Au(111)} \mathrm{also} \mathrm{presents} \mathrm{the} \mathrm{Si}(004)$ peak of the substrate at $2 \theta=82.42^{\circ}$ (not shown). Pole figures of the $\operatorname{Co}\{0002\}$ reflections $\left(2 \theta=51.47^{\circ}\right)$ measured on (b) Co NWs/Pt(111), (c) Co NWs/Co(0001), and (d) Co NWs/Au(111). (e) Schematic representation of a Co NW array on 6-fold symmetry surfaces.

All the above mentioned data indicate that the $\operatorname{Co}(0001)$ crystalline orientation and therefore the vertical growth of Co cylindrical nanocrystals are governed by the symmetry matching between the $\mathrm{Co}(0001)$ growing plane and the different 6-fold symmetry surfaces, whatever the associated lattice mismatches with $\mathrm{Co}(\mathrm{Co} / \mathrm{Pt}=-9.5 \%, \mathrm{Co} / \mathrm{Co}=0, \mathrm{Co} / \mathrm{Au}=-13.1 \%)$. This is consistent with the hexagonal close-packed $(h c p) \operatorname{Co}(0001)$ growth orientation reported in thin film growth studies of $\mathrm{Co} / \mathrm{Pt}(111)^{42,43}$ and $\mathrm{Co} / \mathrm{Au}(111)^{44,45}$ by physical vapor deposition techniques. 
In terms of magnetic properties, magnetic measurements reveal that NW arrays on 6-fold symmetry surfaces exhibit hysteresis loops characteristic of ferromagnetic materials at room temperature. On $\mathrm{Pt}(111)$, Co $\mathrm{NWs} / \mathrm{NRs}$ present a magnetic anisotropy perpendicular to the substrate: the hysteresis loops are more square with higher remnant magnetizations and coercive fields when the magnetic field is applied perpendicular to the metallic film, i.e. parallel to the NRs/NWs (Supporting Fig. S4). This behavior results from the intrinsic shape anisotropy of the NWs/NRs, combined with the uniaxial magnetocrystalline anisotropy along the $\mathrm{Co}[0001]$ direction, and this despite important dipolar interactions. ${ }^{39}$ The association of a high magnetocrystalline anisotropy and a high density is a pre-requisite for application in magnetic recording, therefore the hcp single-crystalline structure is highly desirable for this application. On $\operatorname{Co}(0001)$, despite the similar overall structure to the $\operatorname{Pt}(111)$ which presents an out-of-plane easy axis of the NR array, only a weak magnetic anisotropy is measured along the substrate plane (Supporting Fig. S5). This is attributed to the in-plane contribution of the $20 \mathrm{~nm}$ thick $\mathrm{Co}(0001)$ film on which NRs have grown. An important point concerns the absence of $\mathrm{Co} / \mathrm{Co}$ oxide exchange bias in the low temperature hysteresis loops of the Co NWs/Pt(111) samples preserved from air-exposure during transfer to the magnetometer (Supporting Fig. S4b). This demonstrates that, as in the case of the synthesis of Co nanorods and nanowires in homogeneous conditions, i.e. without substrates, ${ }^{40,41}$ the Co NW growth process intrinsically provides oxygen free Co nano-objects. If samples are air-exposed, a $\mathrm{Co} / \mathrm{Co}$ oxide exchange bias is measured at low temperature and the saturation magnetization slightly decreases. We believe the CoO surface layer passivates the nanowires that retain their metallic core even after long air exposure as confirmed by checking by magnetic measurements at various time intervals (Supporting Fig. S6). 


\section{Co nanowire growth on 4-fold symmetry metallic films}

The same Co growth conditions in the presence of crystalline surfaces of 4-fold symmetry results in different orientations of nanostructures, the growth direction of which are dictated by the substrate.

\section{Co decomposition on $\operatorname{Pt}(001)$}

Figure 4 displays the NR array obtained on a $\mathrm{Pt}(001)$ film after a 3 days reaction at $100^{\circ} \mathrm{C}$ and with a Co/LA/HDA ratio of 1/1.2/1.2. The SEM micrograph of Figure 4a shows tilted NRs with in-plane projections of the NR directions along the $\mathrm{Pt}<110>$ directions of the $\operatorname{Pt}(001)$ film. The TEM cross-section indicates that an intermediate Co layer of around $5 \mathrm{~nm}$ is present between the $\operatorname{Pt}(001)$ film and a layer of slanted NRs (Figure $4 \mathrm{~b})$. The NRs have diameters around $8 \mathrm{~nm}$ and grow along the $h c p \mathrm{Co}[0001]$ axis. Texture measurements by XRD were carried out in order to identify the Co NR orientations. The resulting pole figure of the $\operatorname{Co}\{0002\}$ reflections shown in Figure $4 \mathrm{c}$ reveals 4 satellite spots at $\varphi=45,135,225$ and $315^{\circ}$ indicating that the NRs are oriented along 4 distinct directions and form an angle of $\chi=47.3^{\circ}$ with the normal of the film, i.e. the Pt[001] axis (precise angle measurements are shown in Supporting Fig. S7). The large spot measured in the central area of the pole figure (at $\chi=\varphi^{=} 0^{\circ}$ ) could be due to parasite diffraction of the $\mathrm{MgO}(002)$ and $\mathrm{Pt}(002)$ planes, as $\mathrm{MgO}(002)$ and $\mathrm{Pt}(002)$ diffract at angles close to the $\operatorname{Co}(0002)$ one, and no $\operatorname{Co}(0002)$ peak appears in the XRD $\theta-2 \theta$ diffractogram (Supporting Fig. S8). However, the presence of a limited number of vertical Co NRs on $\operatorname{Pt}(001)$ cannot be totally ruled out. The Co\{0002\} pole figure also indicates that the in-plane projections of the tilted NRs are aligned along the $\mathrm{Pt}<110>$ directions. From the analysis developed above, the NR orientations on $\mathrm{Pt}(001)$ are summarized in Figure 4d. A reaction under conditions corresponding to a nanowire synthesis (Co/LA/HDA ratio of $1 / 2 / 2)$ was also performed on $\mathrm{Pt}(001)$ and also 
leads to tilted NWs with identical spatial orientations to the ones described for NRs (Supporting Fig. S9).
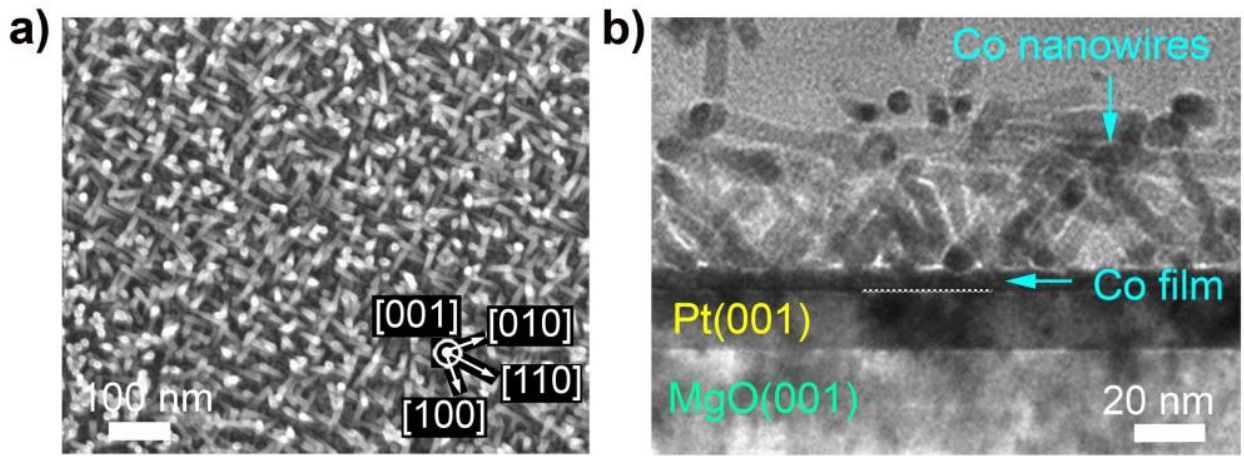

c)

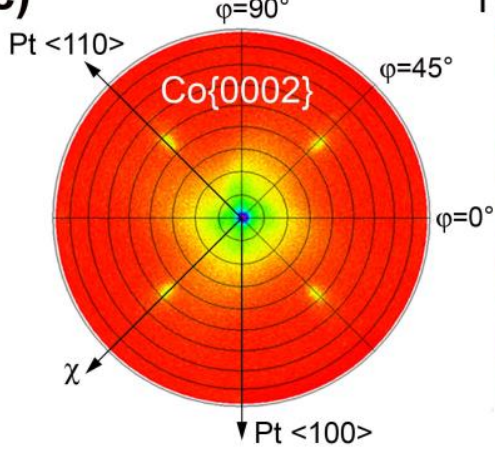

I (ct)

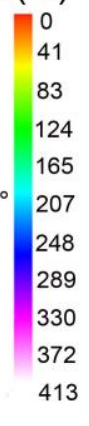

d)

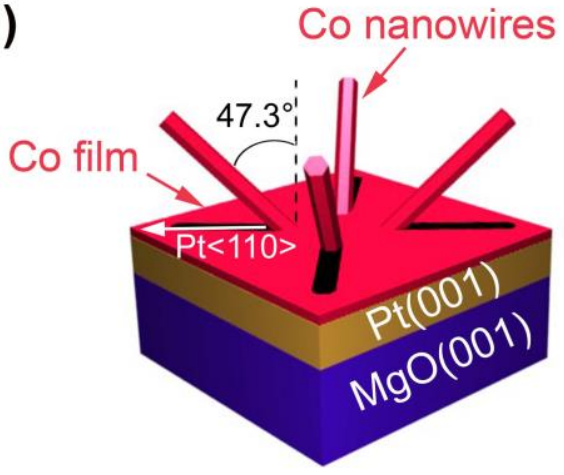

Figure 4. (a) SEM micrograph of a Co NR array grown on $\mathrm{Pt}(001) / \mathrm{MgO}(001)$ (Co/LA/HDA ratio: $1 / 1.2 / 1.2,[\mathrm{Co}]=2.1 \mathrm{mM}, 3.5$ days, $100^{\circ} \mathrm{C}$, solvent: toluene) Inset: crystalline directions of the $\operatorname{Pt}(001)$ film. (b) Cross-sectional TEM micrograph of the same sample. (c) XRD pole figure of the Co $\{0002\}$ reflections, indicating 4 satellite spots at $\chi=47.3^{\circ}$. (d) Schematic 3D view of the Co NR orientations on $\operatorname{Pt}(001)$.

While rationalisation of the growth orientation of Co $\mathrm{NRs} / \mathrm{NWs}$ on $\mathrm{Pt}(001)$ films is not straightforward, an explanation can be given considering the presence of a $5 \mathrm{~nm}$ intermediate Co layer on $\mathrm{Pt}(001)$, in association to the measured tilt angle of the NRs/NWs. We assume that during the first stages of the growth, Co island nucleation occurs on Pt to form the intermediate 
Co layer. From the measured tilt angle of the NWs, it is proposed that Co $h c p$ nuclei grow on $\operatorname{Pt}(001)$ along $\operatorname{Co}(11-23)$ planes as opposed to the growth along $\operatorname{Co}(0001)$ planes on 6-fold symmetry metallic films. Upon growth, these $\operatorname{Co}(11-23)$ islands should exhibit facets composed of (0001) planes in order to minimize their surface energy because such planes are the ones of minimum energy in bulk $h c p$ Co. ${ }^{46}$ Since (0001) and (11-23) planes form an angle of $47.26^{\circ}$ in bulk $h c p \mathrm{Co}$, the (0001) facets of the islands are oriented at $47.26^{\circ}$ from the substrate plane (Supporting Figure S10). As already shown, deposition of Co from the solution on $\operatorname{Co}(0001)$ planes induces a vertical NW growth (Figures 1b, 2d, 3c). Therefore Co deposition on (0001) facets of $\mathrm{Co}(11-23)$ islands results in NW growth perpendicularly to (0001) facets, i.e. along directions at $47.3^{\circ}$ from the normal of the substrate plane, as measured experimentally. In this description, the $\operatorname{Co}(11-23)$ islands would compose the observed $5 \mathrm{~nm}$ thick layer on $\operatorname{Pt}(001)$. Despite the fact that the $\operatorname{Co}(11-23)$ growth orientation on $\operatorname{Pt}(001)$ has never been reported before, whatever the deposition technique, such (11-23) planes would reduce the lattice parameter misfits between Co and Pt lattices (Supporting Fig. S11).

In terms of magnetic properties, the peculiar Co NRs/NWs orientations on $\operatorname{Pt}(001)$ induce strong differences in comparison to vertical Co NRs/NWs on 6-fold symmetry surfaces. As the anisotropy is high along the NW long axis due to the uniaxial Co $h c p$ magnetocrystalline anisotropy, the different NR/NW directions for $\mathrm{NRs} / \mathrm{NW}$ s grown on $\operatorname{Pt}(001)$ induce a dispersion of the magnetic easy axes. Moreover, the $5 \mathrm{~nm}$ Co intermediate layer is expected to favor an inplane anisotropy. Consequently, the out-of-plane direction is a hard axis and a weak anisotropy is observed along the substrate plane (Supporting Fig. S12). Qualitatively, micromagnetic simulations illustrate the influence of the NR orientation distribution and of the Co intermediate layer on the magnetic behavior (Supporting Fig. S13). 


\section{Co decomposition on $\mathrm{Cu}(001)$}

A different $\mathrm{NW}$ configuration is obtained by using a $\mathrm{Cu}(001)$ film as a $2 \mathrm{D}$ seed. After a reaction with a $\mathrm{Co} / \mathrm{LA} / \mathrm{HDA}$ ratio of $1 / 2 / 2$ during $24 \mathrm{~h}$ at $150^{\circ} \mathrm{C}$, the $\mathrm{SEM}$ and $\mathrm{TEM}$ cross-section micrographs also show tilted nanowires on the whole substrate surface (Figure 5a,b respectively). The NW diameter is estimated by TEM to be around $11 \mathrm{~nm}$. TEM observations allow distinguishing two different growth directions (see dashed lines on Figure 5b). As seen in Figure $5 \mathrm{c}$, the $\operatorname{Co}\{0002\}$ pole figure is composed of 8 satellite spots, 4 at $\chi=54.3^{\circ}$ and 4 at $\chi=15.5^{\circ}$ (precise angle measurements in Supporting Fig. S14a-b). As summarized on the 3D scheme given in Figure 5d, it is concluded that there exist 8 directions of tilted NWs, 4 forming an angle of about $15.5^{\circ}$ with the normal of the substrate (i.e. the $\mathrm{Cu}[001]$ axis) and 4 forming an angle of about $54.3^{\circ}$. These 8 directions have projections on the substrate plane that are aligned along the $\mathrm{Cu}<110>$ directions. 

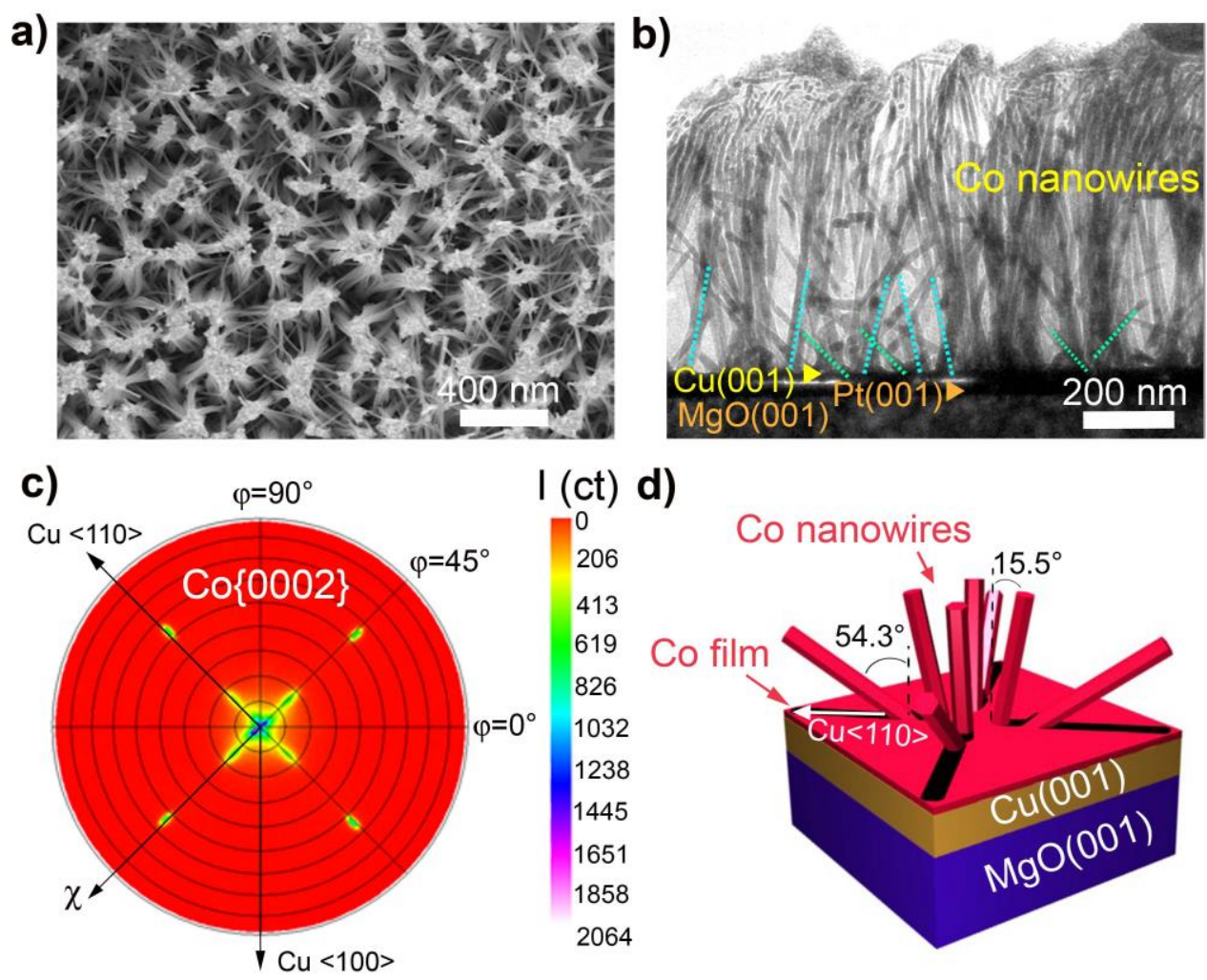

Figure 5. (a) SEM micrograph of a $\mathrm{Co} \mathrm{NW}$ array grown on $\mathrm{Cu}(001) / \mathrm{MgO}(001)(\mathrm{Co} / \mathrm{LA} / \mathrm{HDA}$ ratio: $1 / 2 / 2,[\mathrm{Co}]=5 \mathrm{mM}, 24 \mathrm{~h}, 150^{\circ} \mathrm{C}$, solvent: anisole). (b) Cross-sectional TEM micrographs of the same sample (the dashed lines indicate the two tilted growth directions). (c) XRD pole figure of the Co $\{0002\}$ reflections, indicating 4 satellite spots at $\chi=15.5^{\circ}$ and 4 at $\chi=54.3^{\circ}$. (d) Schematic $3 \mathrm{D}$ view of the $\mathrm{Co} \mathrm{NW}$ orientations on a $\mathrm{Cu}(001)$ film.

The origin of the tilted NW orientations on $\mathrm{Cu}(001)$ can be rationalized as in the Co $\mathrm{NWs} / \mathrm{Pt}(001)$ system. Based on the measured tilt angles, it is suggested that the Co nucleation on the $\mathrm{Cu}(001)$ film induces the growth of face-centered cubic $(f c c)$ Co islands of $\mathrm{Co}(001)$ and $\mathrm{Co}(221)$ orientations (i.e. islands with $\mathrm{Co}(001)$ and $\mathrm{Co}(221)$ planes parallel to $\mathrm{Cu}(001)$, respectively). The $f c c \mathrm{Co}(001)$ growth orientation is commonly reported in Co ultra-thin films deposited on $\mathrm{Cu}(001)$ surfaces by physical vapour deposition techniques. ${ }^{47-49}$ Furthermore, the 
growth of $f c c \mathrm{Co}(001)$ on $\mathrm{Cu}(001)$ is confirmed by XRD measurements (Supporting Fig. S14d). We believe that $f c c \operatorname{Co}(001)$ islands expose facets composed of $\operatorname{Co}(111)$ planes, that is, planes forming an angle of $54.74^{\circ}$ with respect to $\operatorname{Co}(001)$ planes in bulk $f c c$ Co. Since a $f c c \operatorname{Co}(111)$ plane is identical to a $h c p \operatorname{Co}(0001)$ plane in terms of symmetry and interatomic distances, a deposition of Co from the solution on these planes would result to the anisotropic growth of Co and consequently to Co NWs tilted at an averaged measured angle of $54.3^{\circ}$ from the normal of the substrate (see $f c c \operatorname{Co}(001)$ island description in Supporting Fig. S15a). Following the same reasoning, $f c c \mathrm{Co}(221)$ islands could nucleate on $\mathrm{Cu}(001)$ and exhibit facets composed of (111) planes which form an angle of $15.79^{\circ}$ with the (221) planes in bulk $f c c$ Co. Such facets could induce a $\mathrm{Co}[0001]$ growth and therefore the resulting NW long axis at defined directions from the normal of the substrate plane, that is, at $15.5^{\circ}$ as measured experimentally (see $f c c \operatorname{Co}(221)$ island description in Supporting Fig. S15b). To the best of our knowledge, the growth of $f c c$ $\mathrm{Co}(221)$ has never been reported in Co ultra-thin films deposited on $\mathrm{Cu}(001)$ surfaces, whatever the deposition techniques. Nevertheless, it can be shown that the lattice parameter mismatch between $f c c \mathrm{Co}(221)$ and $\mathrm{Cu}(001)$ is the same as between $f c c \mathrm{Co}(001)$ and $\mathrm{Cu}(001)$ (Supporting Fig. S16). A third NW orientation on $\mathrm{Cu}(001)$ can be deduced from the XRD $\mathrm{Co}\{0002\}$ pole figure in Figure 5c. The intense central spot at $\chi=\varphi=0^{\circ}$ could be attributed to vertical NWs because the XRD $\theta-2 \theta$ diffractogram (Supporting Fig. S14d) clearly shows a $h c p \operatorname{Co}(0002)$ peak (or $f c c \operatorname{Co}(111)$ as they diffract at the same angle) which indicates that Co planes of 6-fold symmetry are parallel to the substrate. These NWs may result from Co islands of either $h c p$ $\mathrm{Co}(0001)$ or $f c c \mathrm{Co}(111)$ growth orientation which are also present on $\mathrm{Cu}(001)$, however without an epitaxial relationship with $\mathrm{Cu}(001)$ due to symmetry mismatch. Such islands could then induce a vertical growth of Co NWs. The presence of parasitic Pt(111) crystallites in the Pt 
buffer layer introduced to enhance the crystalline orientation of the $\mathrm{Cu}$ film can moreover favor such a $h c p \operatorname{Co}(0001)$ or $f c c \operatorname{Co}(111)$ growth orientation (Fig. S14d). Contrary to the case of Co $\mathrm{NW} / \mathrm{Pt}(001)$, the presence of a Co intermediate layer at the Co $\mathrm{NW} / \mathrm{Cu}(001)$ interface has not been clearly detected by TEM. However, it has to be noted that the $\mathrm{Co} / \mathrm{Cu}$ interface area is ill defined with disturbed contrasts in TEM, presumably due to a preferential $\mathrm{Cu}$ etching during the cross-sectional sample preparation.

Finally, the growth of Co nanowires has been attempted on commercial thin $\mathrm{Cu}$ foils instead of orientated crystalline films. The results show that the Co NW growth is effective on $50 \mu \mathrm{m}$ thick $\mathrm{Cu}$ foils, with tilted and vertical NWs in less well-defined orientations than for the previous cases due to the polycristallinity of the substrate (Supporting Fig. S17). Such behaviour is consistent with the above mentioned results demonstrating the peculiar link between the NW growth orientations and the crystallographic orientation of the metallic film. The table 1 summarizes the characteristics of the Co NW growth on different substrates.

\begin{tabular}{|l|c|c|c|c|c|c|}
\hline & \multicolumn{2}{|l|}{ 6-fold symmetry surfaces } & \multicolumn{2}{l|}{ 4-fold symmetry surfaces } & $\begin{array}{l}\text { Polycrystalline } \\
\text { textured surface }\end{array}$ \\
\cline { 2 - 7 } & $\operatorname{Pt}(111)$ & $\mathrm{Co}(0001)$ & $\mathrm{Au}(111)$ & $\operatorname{Pt}(001)$ & $\mathrm{Cu}(001)$ & $\mathrm{Cu}$ \\
\hline $\begin{array}{l}\text { NW } \\
\text { orientation }\end{array}$ & vertical & vertical & vertical & tilted $\left(47.3^{\circ}\right)$ & $\begin{array}{l}\text { tilted } \\
\left(15.5^{\circ} \text { and } 54.3^{\circ}\right)\end{array}$ & $\begin{array}{l}\text { vertical and tilted } \\
\left(\sim 15^{\circ} \text { and } \sim 54^{\circ}\right)\end{array}$ \\
\hline $\begin{array}{l}\text { NW diameter } \\
(\mathrm{nm})\end{array}$ & 6 & 8 & 9 & 8 & 11 & - \\
\hline $\begin{array}{l}\text { NW length } \\
(\mathrm{nm})\end{array}$ & 1080 & 1000 & 1300 & - & 700 & - \\
\hline
\end{tabular}

Table 1. Characteristics of the Co NWs grown on different substrates after a similar chemical reaction $\left(\mathrm{Co} / \mathrm{LA} / \mathrm{HDA}\right.$ ratio: $1 / 2 / 2,[\mathrm{Co}]=5 \mathrm{mM}, 24 \mathrm{~h}, 150^{\circ} \mathrm{C}$, solvent: anisole $)$. 


\section{Fe decomposition on $\operatorname{Pt}(001)$ and $\operatorname{Pt}(111)$}

In order to demonstrate the large scope of the solution epitaxial growth method, the same strategy has been applied for the growth of Fe nanostructures. We previously demonstrated that the reduction by $\mathrm{H}_{2}$ of $\left[\mathrm{Fe}\left\{\mathrm{N}\left(\mathrm{SiMe}_{3}\right)_{2}\right\}_{2}\right]$ in solution in the presence of long chain amine and long-chain acid ligands leads to $\mathrm{Fe}$ nanocubes and nanostars. ${ }^{50}$ Under similar experimental conditions and in the presence of $\operatorname{Pt}(111)$ and $\operatorname{Pt}(001)$ surfaces, Fe nanostructures were successfully grown and present patterns which depend on the Pt crystallographic orientation as shown in Figure 6. After $24 \mathrm{~h}$ reaction, the Fe nanostructures on $\mathrm{Pt}(001)$ consist in cubic-like structures (Fig. 6b). On the other hand, the Fe structures on $\mathrm{Pt}(111)$ can be viewed as triangular prisms, i.e. elongated and truncated cubes along $\{110\}$ planes (Fig. 6a). The same characteristic surface features persist after several days of reaction (Supporting Fig. S18). Transmission Electron Microscopy (TEM) of a cross-section for both samples after 3 days of reaction evidence thick body-centered cubic ( $b c c)$ Fe columnar films above the Pt layer of around $80 \mathrm{~nm}$ for $\operatorname{Pt}(111)$ (Fig.6c) and $40 \mathrm{~nm}$ for $\mathrm{Pt}(001)$ (Fig. 6d), suggesting the merging of the nanostructures towards a Fe film. The XRD $\theta-2 \theta$ diffractograms show that Fe grows mainly along $b c c$ Fe(110) on $\mathrm{Pt}(111)$ (Fig. 6e) and along bcc $\mathrm{Fe}(001)$ on $\mathrm{Pt}(001)$ (Fig. 6f), as observed in thin films elaborated by classical vapour-phase methods. ${ }^{51,52}$ The epitaxial growth of Fe on both substrates is illustrated by $\varphi$-scan diagrams on Fe reflections (insets in Fig. 6e, f). Detailed information on the structure and the epitaxial relationships are given in Supporting Fig. S19. Thus, the decomposition of $\mathrm{Fe}$ in the presence of either $\operatorname{Pt}(001)$ or $\operatorname{Pt}(111)$ surfaces leads to nanostructured epitaxial films of metallic $b c c$ Fe, the growth direction being dictated by the Pt exposed surface. The schematic views of Fig. $6 \mathrm{~g}$ and $\mathrm{h}$ illustrate the overall feature of Fe nanostructure orientations obtained on $\mathrm{Pt}(001)$ and $\mathrm{Pt}(111)$ surfaces respectively. As expected, the Fe layers 
obtained after 3 days of reaction are ferromagnetic at room temperature with an in-plane easy axis of magnetization (Supporting Fig. S20).
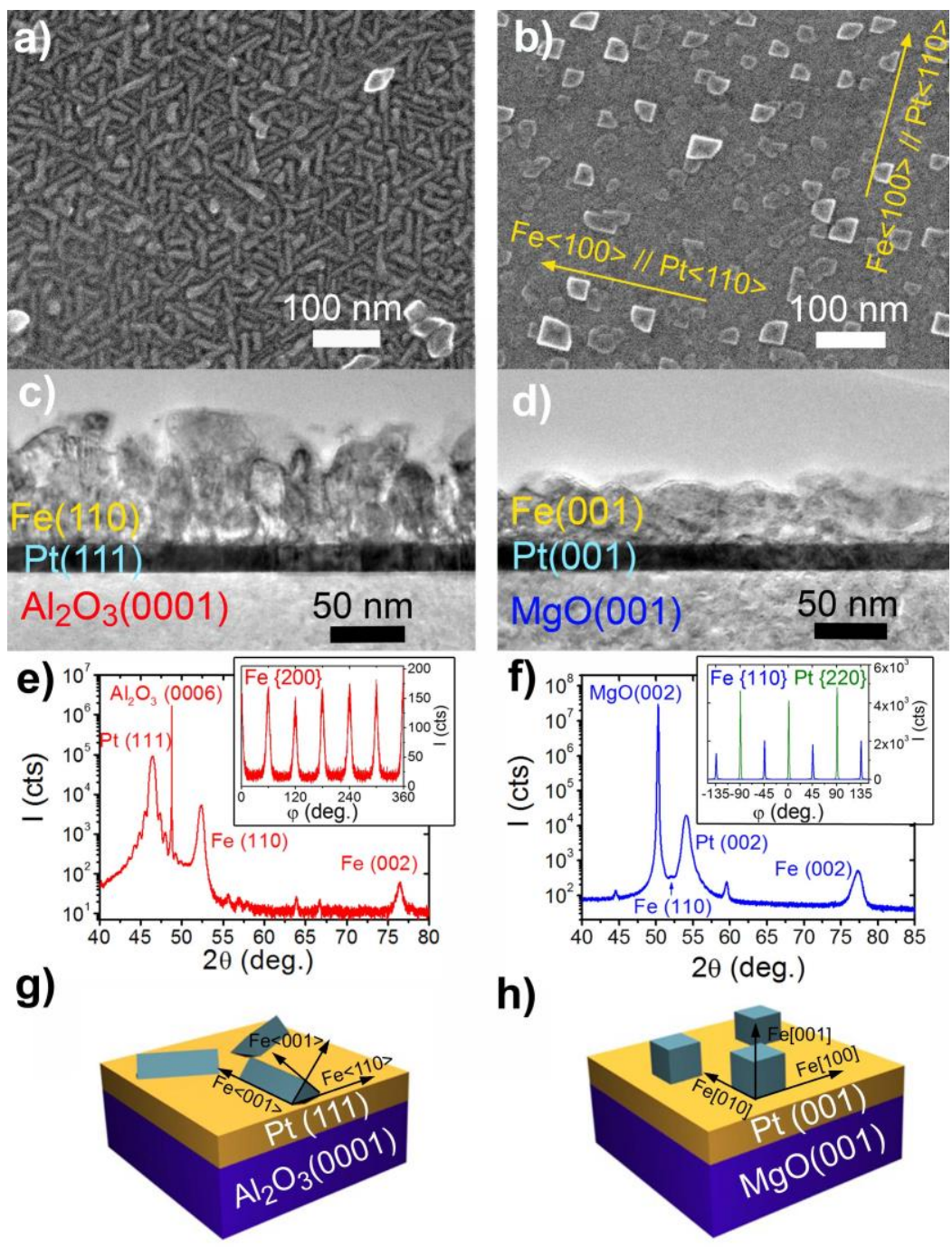

h)

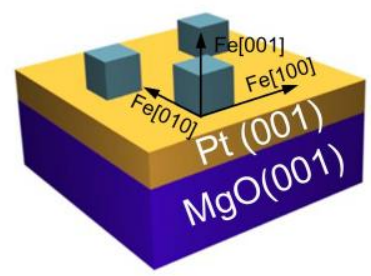

Figure 6. SEM micrographs of Fe nanostructures grown on (a) $\mathrm{Pt}(111)$ and (b) $\mathrm{Pt}(001)$ films, respectively (Fe/LA/HDA: $1 / 2 / 1,[\mathrm{Fe}]=2.5 \mathrm{mM}, 24 \mathrm{~h}, 150^{\circ} \mathrm{C}$, solvent: anisole). (c), (d): TEM crosssection micrographs of the $\mathrm{Fe} / \mathrm{Pt}(111)$ and the $\mathrm{Fe} / \mathrm{Pt}(001)$ growths, respectively (3 days reaction). (e), (f): XRD $\theta-2 \theta$ diffractograms and $\varphi$-scan in inset (3 days reaction). (g), (h): Schematic 3D views of the Fe nanostructure orientations obtained on $\operatorname{Pt}(111)$ and $\operatorname{Pt}(001)$ films respectively before coalescence takes place. 


\section{DISCUSSION}

We have demonstrated that the reduction of a metal complex in solution under mild conditions allows the growth of $\mathrm{Fe}$ and $\mathrm{Co}$ nanostructures on crystalline metallic films. The growth is epitaxial in both cases and the nanostructure orientations are dictated by the crystalline orientation of the film. Concerning Co, on 6-fold symmetry films, we observed a common vertical NR/NW orientation independently of the lattice mismatch. On the other hand, 4-fold symmetry films induce nanowire growth along different orientations. Thus, in all cases the growth process ends-up by forming NRs/NWs similar to those obtained in the absence of any substrate, ${ }^{40,41}$ and this irrespectively of the film characteristics. Concerning Fe, the obtained nanostructures on Pt surfaces can be viewed as merged cubic nanoparticles, truncated along different directions, and with distinct orientations, which depend on the symmetry of the metallic film.

These results allow proposing a general growth mechanism. Before reduction takes place, the metal reservoir consists of several species of different reactivity due to the reaction of the metallic precursor with the amine and acid ligands. ${ }^{40,41}$ The most unstable species are the first to be reduced supplying the first metallic atoms. This first step of heterogeneous nucleation on the film surface leads to nuclei with a crystalline orientation dictated by the symmetry of the surface. As the reaction proceeds, atoms coming from the more stable metal species in solution start to be reduced and add to the nuclei which start to grow. In parallel, upon decomposition of the molecular metallic species, the liberated organic ligands define the size and shape of the islands formed on the surface, by preferential coordination on their less stable facets.

In the case of the formation of free Co NRs, this effect inhibits Co deposition on (10-10) planes which constitutes the wire lateral planes while favoring Co growth along (0001) ones 
which give rise to an anisotropic growth along the $\mathrm{Co}[0001]$ direction. Indeed, free Co nanorods grow along their long axis, that is, by Co addition to $\operatorname{Co}(0001)$ facets, ${ }^{32,33,40}$ whereas in the case of multipods, nanowires of $h c p$ structure grow from $\operatorname{Co}(111)$ facets of $f c c$ seeds. ${ }^{41}$ The present results demonstrate that the same shape-directing function of the organic ligands also acts in the presence of the substrate and consequently leads to an assembly of NWs on the substrate as opposed to a Co film. We suggest that the crystalline orientation of Co nuclei on 6-fold symmetry surfaces is $h c p$ with $\operatorname{Co}(0001)$ growing planes irrespective of the respective lattice parameter mismatches. On 4-fold symmetry surfaces, it is assumed that $f c c$ or $h c p$ Co islands can be stabilized depending on their mismatch with $\mathrm{Cu}(001)$ or $\mathrm{Pt}(001)$ metallic surfaces. Indeed, the appearance of the different growth orientations, i.e. $f c c \mathrm{Co}(001)$ and (221) on $\mathrm{Cu}(001)$ and $h c p$ $\operatorname{Co}(11-23)$ on $\operatorname{Pt}(001)$, could be rationalized in terms of minimized lattice parameter mismatch (Supporting Table S2). It is postulated that the anisotropic Co growth occurs on the 6-fold symmetry facets presented by both $h c p$ and $f c c$ Co islands, that is, on Co(111) facets of $f c c$ Co islands or $\operatorname{Co}(0001)$ facets of $h c p$ ones. This step would require a certain island size and consequently could lead to an intermediate layer of coalesced islands between the NWs and the metallic film before NRs/NWs start to appear, as for Co NWs/Pt(001). Once the anisotropic growth is engaged on specific facets, it is pursued on $\mathrm{Co}(0001)$ planes, and adopting the shape imposed by the solution.

In this description, the $\mathrm{Fe}$ nucleation on metallic surfaces also leads to nuclei with crystallographic orientations dictated by the surface. In the absence of a substrate, the growth of Fe nanocrystals in solution leads to $b c c$ Fe cubes or concave cubes with ligands acting as shapedirecting agents. ${ }^{50}$ In the presence of a substrate, we postulate that the ligands play the same directing role, as observed in solution. The growth mode of Fe nanostructures with cubic 
symmetry implies a $\mathrm{Fe}<100>$ preferential growth direction which is not a strictly anisotropic growth direction as opposed to Co NR/NW. As both $\mathrm{Fe}(110)$ and $\mathrm{Fe}(100)$ orientations obtained on $\operatorname{Pt}(111)$ and $\operatorname{Pt}(001)$ respectively possess a $<100>$ direction along the film plane (Fig. 6g,h), the Fe initial nanostructures on Pt grow also along the substrate plane. Consequently, as the reaction proceeds, the $\mathrm{Fe}$ nanostructures continue to grow on $\mathrm{Pt}$ and finally merge together to give a nanostructured film.

Despite the fact that several points remain unclear for the moment (differences in NR/NW diameters, different Fe film thickness at long reaction time), our results suggest that the solution finally imposes its natural tendency by (i) allowing a pre-structuration of the substrate film by epitaxial nucleation of islands and (ii) selecting island facets from which the growth can take place as dictated by its composition. This is the key point of the present results suggesting that the growth of shape-controlled nanocrystals, well-controlled in solution phase syntheses, can operate on appropriate 2D seeds and proceed via self-organization of the nano-objects on a planar device. This concept is of fundamental interest because it means that the different nanocrystals reported in the literature and synthesized by wet chemical methods could in principle be grown on suitable films. In the case of anisotropic nanocrystals, such as Co $\mathrm{NRs} / \mathrm{NWs}$, this approach is particularly attractive, illustrating the efficient coupling between the intrinsic Co anisotropic crystal lattice and specific organic ligand coordination leading to different growth possibilities, tilted or not, depending on the substrate used. Extension of the method to other anisotropic nanocrystals synthesized by solution phase syntheses will open interesting new bottom-up approaches for nanotechnology. 


\section{CONCLUSION}

Here we report the detailed general approach of a solution phase synthesis of purely metallic nano-objects on various metallic surfaces of different crystalline symmetries. The most interesting system, which benefits from the Co intrinsic anisotropic growth, concerns the Co NRs/NWs growth on surfaces by decomposition of a Co coordination precursor in the presence of acid and amine ligands. Indeed, the use of 6-fold symmetry substrates of various metals yields Co NWs grown perpendicularly to the substrate surface, without any exception. On the other hand, 4-fold symmetry substrates give rise to nanowires that grow at well-defined, albeit different orientations depending on the epitaxial relationships and the lattice mismatch between the substrate and the nucleating Co islands. The case of Fe confirms the generality of the approach and shows that as expected, if the nanocrystal growth is not strictly anisotropic, the obtained nanostructures tend to merge together upon growth, at longer reaction times, to result in a nanostructured film instead of an array of distinct nano-objects.

These results have no precedent using physical deposition methods. We previously presented the interesting magnetic properties of ultra-dense arrays of NWs on $\operatorname{Pt}(111)$, particularly interesting in the field of future magnetic recording where the high dipolar interactions experienced at ultra-high densities can be overcome thanks to the uniaxial magnetocrystalline anisotropy of the single-crystalline $h c p$ NWs to result in a perpendicular magnetic anisotropy. ${ }^{39}$ On the other hand, the growth of tilted NWs on 4-fold symmetry metallic films represents an original approach to support non-aggregated 1D catalysts on a substrate. The use of fully conductive, ductile and inexpensive substrates such as thin $\mathrm{Cu}$ foil is particularly attractive. In addition, considering the larger scope of metallic 1D nanocrystals, by adapting the solution syntheses of free metallic NWs reported in the literature to induce their heterogeneous nucleation 
and growth on a substrate, the solution epitaxial growth method could constitute an alternative to the VLS method for the growth of metallic NWs on surfaces. Finally, thanks to the ability of chemical approaches to induce the epitaxial growth of various materials on pre-synthesized nanocrystals, ${ }^{36}$ one can envisage to modify the nanowires of an array through subsequent deposition steps of other materials towards more complex nanostructures and multifunctional materials of specific properties.

\section{METHODS}

Thin film fabrication. The crystalline metallic films were grown in a vacuum chamber $\left(3 \times 10^{-8}\right.$ mbar) connected to a glove box in order to avoid any oxygen or water contamination when passing from physical vapour deposition to chemical solution growth. Before film deposition, the $10 \mathrm{~mm} \times 10 \mathrm{~mm} \times 1 \mathrm{~mm} \alpha-\mathrm{Al}_{2} \mathrm{O}_{3}(0001)$ sapphire and $\mathrm{Mg} 0(001)$ substrates (furnished by Neyco) were annealed at $750{ }^{\circ} \mathrm{C}$ for an hour under high vacuum, in order to clean the surface. The 20nm thick films were deposited using thermal evaporation or Direct Current (DC) magnetron targets at $5 \times 10^{-3}$ mbar Ar pressure. Details about the deposition conditions of the different epitaxial films are listed in the Supporting Table 1 (temperature, buffer layer, etc). Xray diffraction $(\mathrm{XRD})$ and $\mathrm{X}$-ray reflectivity $(\mathrm{XRR})$ measurements were performed in order to control the growth orientation and epitaxy, the thickness and the roughness degree. The $10 \mathrm{~mm} \times 10 \mathrm{~mm} \times 50 \mu \mathrm{m}$ commercial $\mathrm{Cu}$ foils were purchased from Neyco. They were conditioned in individual oxygen-free sachets and kept in the glove-box. They were opened immediately prior to use and immersed in the growth solution without any further treatment.

Co nanowire growth. All solutions were prepared in a glove-box, toluene was distilled, degassed by three freeze-pump-thaw cycles and kept in the glove-box under activated molecular 
sieves (Acros-Organics 4A 8-12 Mesh) to remove traces of water. Anhydrous anisole was purchased from Aldrich $(99,7 \%)$ and kept in the glove-box under activated molecular sieves in order to remove traces of water. The metal precursor $\left[\mathrm{Co}\left\{\mathrm{N}\left(\mathrm{SiMe}_{3}\right)_{2}\right\}_{2}(\right.$ thf $\left.)\right]$ was furnished by NanoMePS. Hexadecylamine (HDA) (Aldrich) and lauric acid (LA) (Acros) were kept in the glove box and used as received.

In a representative reaction resulting in the growth of long nanowires, a solution of $\left[\mathrm{Co}\left\{\mathrm{N}\left(\mathrm{SiMe}_{3}\right)_{3}\right\}_{2}\right.$ (thf) $](22.6 \mathrm{mg}, 0.05 \mathrm{mmol})$, in $2 \mathrm{ml}$ of anisole is rapidly added to a mixture of HDA (24.2 $\mathrm{mg}, 0.10 \mathrm{mmol})$ and LA(20.0 $\mathrm{mg}, 0.10 \mathrm{mmol})$ in $8 \mathrm{ml}$ of anisole (Co/LA/HDA molecular ratio $=1 / 2 / 2,[\mathrm{Co}]=5 \mathrm{mM})$. The solution is introduced in a Fischer-Porter pressure bottle. The substrate is immersed in the solution with the metallic film facing the bottom of the bottle. The Fischer-Porter is removed from the glove-box, the Ar is evacuated and the bottle is charged with $\mathrm{H}_{2}$ to 3 bars. The solution containing the substrate is heated to $150^{\circ} \mathrm{C}$ for $24 \mathrm{~h}$. At the end of the reaction the solution is cooled down and the Fischer-Porter transferred to the glove box. A TEM grid is prepared by drop-casting some drops of the supernatant solution. At the end of the reaction, the solution is cooled down with cold water and transferred into the glove box. The substrate is removed from the solution and washed 3 times with toluene, then washed twice with a solution of HDA in THF (tetrahydofurane) $(10 \mathrm{mg} / \mathrm{mL}$ ), assisted by ultra sounds during 1 minute, and finally washed in toluene. The substrate was then observed by SEM. The same procedure is used in all experiments.

The same procedure is followed for the formation of nanorods, by adjusting the amounts of reactants so that $\mathrm{Co} / \mathrm{LA} / \mathrm{HDA}=1 / 1.2 / 1.2$. and $[\mathrm{Co}]=2.1 \mathrm{mM}$, and by performing the reaction at $100^{\circ} \mathrm{C}$ for longer reaction times. Toluene was used instead of anisole for the reactions performed at $100^{\circ} \mathrm{C}$, unless otherwise stated. The specific reaction conditions (concentrations, temperature, 
reaction times) for each case are mentioned in the text and/or in the corresponding Figure captions.

Iron growth. A solution of $\left[\mathrm{Fe}\left\{\mathrm{N}\left(\mathrm{SiMe}_{3}\right)_{2}\right\}_{2}\right](9.4 \mathrm{mg}, 0.012 \mathrm{mmol})$, in $2 \mathrm{ml}$ of anisole is rapidly added to a mixture of HDA $(6.0 \mathrm{mg}, 0.025 \mathrm{mmol})$ and LA $(10.0 \mathrm{mg}, 0.050 \mathrm{mmol})$ in $8 \mathrm{ml}$ of anisole $(\mathrm{Fe} / \mathrm{LA} / \mathrm{HDA}=1 / 2 / 1,[\mathrm{Fe}]=2,5 \mathrm{mM})$. The solution is introduced in a Fischer-Porter pressure bottle. The substrate is then immersed in the solution with the Pt layer facing the bottom of the bottle. The Fischer-Porter is removed from the glove-box and charged with $\mathrm{H}_{2}$ to 3 bars during $7 \mathrm{~min}$. The reaction is heated to $150^{\circ} \mathrm{C}$ for $24 \mathrm{~h}$ or 3 or 4 days. At the end of the reaction the solution is cooled down and the Fischer-Porter transferred to the glove box. The substrate is washed with toluene in order to remove the organic surfactants and observed by FEG-SEM. A TEM grid is prepared by drop-casting some drops of the transparent supernatant solution in order to verify whether homogeneous growth has taken place in solution.

Electron microscopy (SEM and TEM). The FEG-SEM (Field Emission Gun-Scanning Electron Microscopy) observations were carried out on a JEOL JSM6700F. Samples for crosssectional TEM analysis were prepared following the standard method: the samples were first mechanically polished down to about 10 micron thickness, then the final thinning to electron transparency was achieved by ion milling at low angle $\left(7^{\circ}\right)$ and low voltage $(5 \mathrm{kV})$ using a Precision Ion Polishing System (PIPS from Gatan). The TEM samples were investigated using a Tecnai F20 fitted with a Cs corrector (CEOS) which point resolution is $0.12 \mathrm{~nm}$.

X-ray Diffraction (XRD). The X-ray diffraction measurements were performed on a $\theta-2 \theta$ 4circles PANalitycal Empyrean diffractometer using a Co tube and $\mathrm{Ge}(220)$ monochromator to 
select $\mathrm{Co} \mathrm{K} \alpha_{1}$ radiation $\left(\lambda_{\mathrm{K} \alpha 1} \mathrm{Co}=1.78901 \AA\right)$. The different axes of the diffractometer are displayed on Supporting Figure S3. Each pole figure is recorded during approximately $60 \mathrm{~h}$.

Supporting Information Available: Details about thin film fabrication, definition of the angle during X-ray diffraction experiments, TEM micrograph of Co NWs/Au(111), SEM micrographs of Co NRs/Pt(111) and Co NRs/Co(0001), complementary XRD datas of Co NWs/Pt(001) and Co $\mathrm{NWs} / \mathrm{Cu}(001)$, schematic illustrations of $h c p \operatorname{Co}(11-23)$ islands on $\mathrm{Pt}(001), f c c \operatorname{Co}(221)$ islands on $\mathrm{Cu}(001)$ and $f c c \mathrm{Co}(001)$ islands on $\mathrm{Cu}(001)$, lattice parameter mismatches between $f c c \mathrm{Co}(001), f c c \mathrm{Co}(221), h c p \operatorname{Co}(11-23)$ and $\mathrm{Cu}(001)$ and $\mathrm{Pt}(001)$ films respectively, SEM and XRD data of Co NWs grown on Cu foils, SEM micrographs of Fe nanostructures on Pt(111) and $\mathrm{Pt}(001)$ films after long reaction times, two-dimensional surface lattice configurations of Fe on $\operatorname{Pt}(001)$ and $\mathrm{Pt}(111)$, magnetic hysteresis loops of Co NRs/Pt(111), Co NWs/Pt(111), Co NRs/Co(0001), Co NRs/Pt(001) and Fe nanostructured films on $\operatorname{Pt}(111)$ and $\operatorname{Pt}(001)$. This material is available free of charge via the Internet at http://pubs.acs.org.

\section{AUTHOR INFORMATION}

Corresponding Author

*Blon Thomas, thomas.blon@insa-toulouse.fr

* Katerina Soulantica, ksoulant@insa-toulouse.fr

Present Addresses

$\dagger$ Nikos Liakakos, nliakakos@ lbl.gov, +1-510-486-4251, Chemical Sciences Division, Lawrence Berkeley National Laboratory, 1 Cyclotron Road, Berkeley, California 94720, United States.

$\dagger$ Benoit Cormary, benoit.cormary@lcc-toulouse.fr, +33 5613331 39, CNRS, LCC (Laboratoire de Chimie de Coordination), 205 route de Narbonne, 31077 Toulouse Cedex 4, Université de Toulouse, UPS, INPT, 31077, Toulouse, France. 
Conflict of Interest: The authors declare no competing financial interest.

\section{ACKNOWLEDGMENTS}

The authors thank the ANR for the BATMAG project (ANR-07-BLAN-0296), the DENSAR project (ANR-14-CE07-0025-01), the European Commission for the FP7 NAMDIATREAM project (EU NMP4-LA-2010-246479), the European Commission and the Région Midi-Pyrénées for the POCTEFA Interreg project (MET-NANO EFA 17/08), the European Commission, FEDER and the Région Midi-Pyrénées for NANOBAT I and II projects and the TEMSCAN service for the SEM.

\section{ABBREVIATIONS}

VLS vapor-liquid-solid; NRs nanorods; NWs nanowires; LA lauric acid; HDA hexadecylamine ; SEM scanning electron microscope; TEM transmission electron microscopy ; XRD X-ray diffraction; CVD chemical vapor deposition; $h c p$ hexagonal close-packed; $f c c$ face-centered cubic; bcc body-centered cubic.

\section{REFERENCES}

${ }^{1}$ Gao, Y.; Tang, Z. Design and Application of Inorganic Nanoparticle Superstructures: Current Status and Future Challenges. Small 2011, 7, 2133-2146

2 Grzelczak, M.; Vermant, J.; Furst, E. M.; Liz-Marzan, L. M. Directed Self-Assembly of Nanoparticles. ACS Nano 2010, 4, 3591-3605 
${ }^{3}$ Chen, Q.; Chul Bae, S.; Granick, S. Directed Self-Assembly of a Colloidal Kagome Lattice. Nature 2011, 469, 381-384

${ }^{4}$ Vigderman, L.; Khanal, B. P.; Zubarev, E. R. Functional Gold Nanorods: Synthesis, SelfAssembly, and Sensing Applications. Adv. Mater. 2012, 24, 4811-4841

${ }^{5}$ Li, L.; Seng, K. H.; Chen, Z.; Guo,Z.; Liu, H. K. Self-Assembly of Hierarchical Star-like Co3O4 Micro/Nanostructures and their Application in Lithium Ion Batteries. Nanoscale 2013, 5, $1922-1928$

${ }^{6}$ Whitesides, G. M.; Grzybowski, B. Self-Assembly at all Scales. Science 2002, 295, 2418-2421

7 Busseron, E.; Ruff, Y.; Moulin, E.; Giuseppone, N. Supramolecular Self-Assemblies as Functional Nanomaterials. Nanoscale 2013, 5, 7098-7140

8 Kwiat, M.; Cohen, S.; Pevzner, A.; Patolsky, F. Large-Scale Ordered 1D-Nanomaterials Arrays: assembly or not? Nano Today 2013, 8, 677-694

9 Nie, Z.; Petukhova, A.; Kumacheva, E. Properties and Amerging Applications of SelfAssembled Structures Made from Inorganic Nanoparticles. Nat. Nanotechnol. 2010, 5, 15-25

${ }^{10}$ Amatore, C. Is there an Intrinsic Limit to the Size of 2D Supracrystals Built from Weakly Interacting Nanoparticles? Chem. - Eur. J. 2008, 14, 8615-8623

11 Greene, L. E.; Yuhas, B. D.; Law, M.; Zitoun, D.; Yang, P. Solution-Grown Zinc Oxide Nanowires. Inorg. Chem. 2006, 45, 7535-7543 
12 Wagner, R. S.; Ellis, W. C. Vapor-Liquid-Solid Mechanism of Single Crystal Growth. Appl. Phys. Lett. 1964, 4, 89-90

${ }^{13}$ Wang, N.; Cai, Y.; Zhang, R. Q. Growth of Nanowires. Mater. Sci. Eng., R 2008, 60, 1-51

14 Adhikari, H.; Marshall, A. F.; Chidsey, C. E. D.; McIntyre, P. C. Germanium Nanowire Epitaxy: Shape and Orientation Control. Nano Lett. 2006, 6, 318-323

15 Lugstein, A.; Steinmair, M.; Hyun, Y. J.; Hauer, G.; Pongratz, P.; Bertagnolli, E. PressureInduced Orientation Control of the Growth of Epitaxial Silicon Nanowires. Nano Lett. 2008, 8, 2310-2314

16 Vayssieres, L. An Aqueous Solution Approach to Advanced Metal Oxide Arrays on Substrates. Appl. Phys. A: Mater. Sci. Process. 2007, 89, 1-8

${ }^{17}$ Appell, D. Wired for Success. Nature 2002, 419, 553-555

18 Martin, C. R. Nanomaterials: a Membrane-Based Synthetic Approach. Science 1994, 266, 1961-1966

${ }^{19}$ Lee, W.; Park, S.-J. Porous Anodic Aluminum Oxide: Anodization and Templated Synthesis of Functional Nanostructures. Chem. Rev. 2014, 114, 7487-7556

20 Thurn-Albrecht, T.; Schotter, J.; Kästle, G. A.; Emley, N.; Shibauchi, T.; Krusin-Elbaum, L.; Guarini, K.; Black, C. T.; Tuominen, M. T.; Russell, T. P. Ultrahigh-Density Nanowire Arrays Grown in Self-Assembled Diblock Copolymer Templates. Science 2000, 290, 2126-2129 
21 Brumlik, C. J.; Menon, V. P.; Martin, C. R. Template Synthesis of Metal Microtubule Ensembles Utilizing Chemical, Electrochemical, and Vacuum Deposition Techniques. J. Mater. Res. 1994, 9, 1174-1183

${ }^{22}$ Huczko, A. Template-Based Synthesis of Nanomaterials. Appl. Phys. A: Mater. Sci. Process. 2000, $70,365-376$

${ }^{23}$ Zhang, X. Y.; Xu, L. H.; Dai, J. Y.; Chan, H. L. W. Fabrication and Magnetic Behavior of CoNi Nanowire Arrays with Small Diameters. Phys. B (Amsterdam, Neth.) 2004, 353, 187-191

${ }^{24}$ Darques, M.; Encinas, A.; Vila, L.; Piraux, L. Controlled Changes in the Microstructure and Magnetic Anisotropy in Arrays of Electrodeposited Co Nanowires Induced by the Solution $\mathrm{pH}$. J. Phys. D: Appl. Phys. 2004, 37, 1411-1416

25 Vidal, F. ; Zheng, Y. ; Schio, P.; Bonilla, F. J. ; Barturen, M. ; Milano, J. ; Demaille, D. ; Fonda, E. ; de Oliveira, A. J. A. ; Etgens, V. H. Mechanism of Localization of the Magnetization Reversal in 3 nm Wide Co Nanowires. Phys. Rev. Lett. 2012, 109, 117205

26 Jamet, M.; Barski, A.; Devillers, T.; Poydenoet, V.; Dujardin, R. ; Bayle-Guillemaud, P. ; Rothman, J.; Bellet-Amalric, E.; Marty, A.; Cibert, J.; et al. High-Curie-Temperature Ferromagnetism in Self-Organized Ge ${ }_{1-x} \mathrm{Mn}_{\mathrm{x}}$ Nanocolumns. Nat. Mater. 2006, 5, 653-659

${ }^{27}$ Mohaddes-Ardabili, L.; Zheng, H.; Ogale, S. B. ; Hannoyer, B.; Tian, W.; Wang, J.; Lofland, S. E.; Shinde, S. R.; Zhao, T.; Jia, Y.; et al. Self-Assembled Single-Crystal Ferromagnetic Iron Nanowires Formed by Decomposition. Nat. Mater. 2004, 3, 533-538 
${ }^{28}$ Kim, S-I.; Yoon, H.; Lee, H.; Lee, S.; Jo, Y.; Lee, S.; Choo, J.; Kim, B. Epitaxy-Driven Vertical Growth of Single-Crystalline Cobalt Nanowire Arrays by Chemical Vapor Deposition. J. Mater. Chem. C 2015, 3, 100-106

29 Yao, J.; Liu, Z.; Liu, Y.; Wang, Y.; Sun, C.; Bartal, G.; Stacy, A. M.; Zhang, X. Optical Negative Refraction in Bulk Metamaterials of Nanowires. Science 2008, 32 1, 930

${ }^{30}$ Liu, Q.; Cui, Y.; Gardner, D. ; Li, X. ; He, S.; Smalyukh, I. I. Self-Alignment of Plasmonic Gold Nanorods in Reconfigurable Anisotropic Fluids for Tunable Bulk Metamaterial Applications. Nano Lett. 2010, 10, 1347-1353

31 Terris, B. D.; Thomson, T. Nanofabricated and Self-Assembled Magnetic Structures as Data Storage Media. J. Phys. D: App. Phys. 2005, 38, R199-R222

32 Dumestre, F.; Chaudret, B.; Amiens, C.; Respaud, M.; Fejes, P. Unprecedented Crystalline Super-Lattices of Monodisperse Cobalt Nanorods. Angew. Chem., Int. Ed. 2003, 42, 5213 -5216

${ }^{33}$ Dumestre, F.; Chaudret, B.; Amiens, C.; Fromen, M-C.; Casanove, M-J.; Renaud, P.; Zurcher, P. Shape Control of Thermodynamically Stable Cobalt Nanorods Through Organometallic Chemistry. Angew. Chem., Int. Ed. 2002, 41, 4286-4289

34 Heng, H.; Gibbons, P. C.; Kelton, K.F.; Buhro, W. E. Heterogeneous Seeded Growth: A Potentially General Synthesis of Monodisperse Metallic Nanoparticles. J. Am. Chem. Soc. 2001, $123,9198-9199$

35 Carbone, L.; Cozzoli, P. D. Colloidal Heterostructured Nanocrystals: Synthesis and Growth Mechanisms. Nano Today 2010, 5, 449-493 
${ }^{36}$ Liakakos, N.; Gatel, C.; Blon, T.; Lentijo-Mozo, S.; Altantzis, T.; Garcia-Martelot, C.; Lacroix, L.-M.; Respaud, M.; Bals, S.; Van Tendeloo, G.; et al. Co-Fe Nanodumbbells : Synthesis, Structure and Magnetic Properties. Nano Lett. 2014, 14, 2747-2754

${ }^{37}$ Habas, S. E.; Lee, H.; Radmilovic, V.; Somorjai, G. A.; Yang, P. Shaping Binary Metal Nanocrystals Through Epitaxial Seeded Growth. Nat. Mater. 2007, 6, 692-697

${ }^{38}$ Sneed, B. T.; Kuo, C-H.; Brodsky, C. N.; Tsung, C-K. Iodide-Mediated Control of Rhodium Epitaxial Growth on Well-Defined Noble Metal Nanocrystals: Synthesis, Characterization, and Structure-Dependent Catalytic Properties. J. Am. Chem. Soc. 2012, 134, 18417-18426

${ }^{39}$ Liakakos, N.; Blon, T.; Achkar, C.; Vilar, V.; Cormary, B.; Tan, R.P.; Benamara, O.; Chaboussant, G.; Ott, F.; Warot-Fonrose, B. et al. Solution Epitaxial Growth of Cobalt Nanowires on Crystalline Substrates for Data Storage Densities Beyond 1 Tbit/in². Nano Lett. 2014, $14,3481-3486$

${ }^{40}$ Wetz, F.; Soulantica, K.; Respaud, M.; Falqui, A.; Chaudret, B. Synthesis and Magnetic Properties of Co Nanorod Superlattices. Mater. Sci. Eng., C 2007, 27, 1162-1166

${ }^{41}$ Liakakos, N.; Cormary, B.; Li, X.; Lecante, P.; Respaud, M.; Maron, L.; Falqui, A.; Genovese, A.; Vendier, L.; Koïnis, S.; et al. The Big Impact of a Small Detail: Cobalt Nanocrystal Polymorphism as a Result of Precursor Addition Rate During Stock Solution Preparation. J. Am. Chem. Soc. 2012, 134, 17922-17931 
42 Thiele, J.; Belkhou, J. R.; Bulou, H.; Heckmann, O.; Magnan, H.; Le Fèvre, P.; Chandesris, D.; Guillot, C. EXAFS Study of the Crystallographic Structure of Cobalt Thin Films on Pt(111). Surf. Sci. 1997, 384, 120-128

${ }^{43}$ Grütter, P.; Dürig, U. T. Growth of Vapor Deposited Cobalt Films on Pt(111) Studied by Scanning Tunneling Microscopy. Phys. Rev. B: Condens. Matter Mater. Phys. 1994, 49, 20212029

${ }^{44}$ Voigtlander, B.; Meyer, G.; Amer, N. M.; Epitaxial Growth of Thin Magnetic Cobalt Films on Au(111) Studied by Scanning Tunneling Microscopy. Phys. Rev. B: Condens. Matter Mater. Phys. 1991, 44, 10354

${ }^{45}$ Ardhuin, H.; Snoeck, E.; Casanove, M.J. Epitaxial Growth of Magnetic Au/Co/Au Sandwiches Studied by TEM. J. Cryst. Growth 1997, 182, 394-402

46 Vitos, L.; Ruban, A. V.; Skriver, H. L.; Kollár, J. The Surface Energy of Metals. Surf. Sci. 1998, 411, 186-202

${ }^{47}$ Li, H.; Tonner, B. P. Structure and Growth Mode of Metastable $f c c$ Cobalt Ultrathin Films on $\mathrm{Cu}(001)$ as Determined by Angle-Resolved X-Ray Photoemission Scattering. Surf. Sci. 1990, $237,141-152$

${ }^{48}$ Heinrich, B.; Cochran, J.F.; Kowalewski, M.; Kirschner, J.; Celinski, Z.; Arrott, A. S.; Myrtle, K. Magnetic Anisotropies and Exchange Coupling in Ultrathin fcc $\operatorname{Co}(001)$ Structures. Phys. Rev. B: Condens. Matter Mater. Phys. 1991, 44, 9348-9361 
49 Turko, D.; Morawski, I.; Nowicki, M. Growth and Crystalline Structure of Co Layers on Cu(001). Appl. Surf. Sci. 2008, 254, 4391-4395

${ }^{50}$ Lacroix, L.-M.; Lachaize, S.; Falqui, A.; Respaud, M.; Chaudret, B. Iron Nanoparticle Growth in Organic Superstructures, J. Am. Chem. Soc. 2009, 131, 549-557

51 Chen, Y.-J.; Chang, C.-C.; Ho, H.-Y.; Tsay, J.-S. Effects of Interfacial Structure on the Magnetic Properties of Ultrathin Fe/Pt(111) Films with Ag Buffer Layer, Thin Solid Films 2011, $519,8343-8346$

52 Hufnagel, T.C.; Kautzky, M.C.; Daniels, B.J.; Clemens, B.M. Structural Evolution During Deposition of Epitaxial Fe/Pt(001) Multilayers, J. Appl. Phys. 1999, 86, 2609-2616. 